\section{Management of concussion in disability sport: a different ball game?}

\author{
Liam Richard West, ${ }^{1}$ Steffan Griffin, ${ }^{2}$ Richard Weiler, ${ }^{3,4,5}$ \\ Osman Hassan Ahmed ${ }^{5,6}$
}

Concussion management in sport is a serious medical issue. Frequent highprofile incidents coupled with ongoing debate and research surrounding the definition, diagnosis and management of concussion mean that it is likely to remain a hot topic. ${ }^{1}$ Internationally, concussion has become a key focus for many sporting governing bodies, with a range of educational campaigns aimed at improving recognition and management. ${ }^{2}$

\section{CONCUSSION IN SPECIAL POPULATIONS}

Football is the world's most popular global sport, and appropriately many disability football leagues have been developed to provide competitive opportunities for those individuals with disability wishing to compete outside 'mainstream' football. Adapted versions of football for athletes with major disabilities including learning disability; visual impairment; cerebral palsy/acquired brain injury; hearing impairment and amputation are all in existence. There has been a suggestion of an increased risk of musculoskeletal injury and head injury from participation, including concussion. ${ }^{3}$

However, at present the rates of and best-practice assessment and management for concussion in these formats of football are unknown. ${ }^{4}$

\section{CONCUSSION ASSESSMENT AND MANAGEMENT}

Building on the previous versions of the Sports Concussion Assessment Tool (SCAT), the SCAT3 has helped sports medicine professionals to diagnose and manage concussion, providing guidelines on which an individual management protocol can be based. The 2012 Zurich

${ }^{1}$ Royal Melbourne Hospital, Melbourne, Victoria, Australia; ${ }^{2}$ College of Medical and Dental Sciences, University of Birmingham, Birmingham, UK; ${ }^{3}$ University College London Hospitals NHS Foundation Trust \& UCL Institute of Sport, Exercise and Health, London, UK; ${ }^{4}$ Fortius Clinic, London, UK; ${ }^{5}$ FA Centre for Disability Football Research, The Football Association, BurtonUpon-Trent, UK; ${ }^{6}$ Faculty of Health and Social Sciences, Bournemouth University, Bournemouth, UK

Correspondence to Dr Liam Richard West, Royal Melbourne Hospital, Gratton Street, Parkville, Melbourne, VIC, Australia; liamwestsem@hotmail.co.uk
Consensus Meeting generated the Child SCAT3 for use in athletes aged 5-12 years to help address the controversial issue of diagnosis and management of paediatric concussion. Makdissi and colleagues ${ }^{5}$ proposed that children and non-elite individuals with limited resources should be managed more conservatively than elitelevel athletes, in line with modifications made to the SCAT3.

Given these adaptations for the paediatric population, it is reasonable to suggest that specific guidelines for athletes with disability should also be generated. Ahmed and colleagues highlighted the limited evidence base underpinning sports medicine in disability football, ${ }^{6}$ and the paucity of evidence for assessing and managing concussion in disabled athletes warrants further investigation.

\section{CP WORLD CUP EXPERIENCE}

The most recent Cerebral Palsy (CP) Football World Championships were held at St. Georges Park, England, in June 2015. As with all disability sport, athletes must pass a strict neurological condition criteria test before they are eligible for CP football. During this event, informal discussions with the head of medical services of the 15 competing countries revealed their current approaches to dealing with concussion in elite CP football.

The majority of clinicians (eight physiotherapists, and six doctors, with an average of 4 years experience in the role) reported receiving some form of education regarding concussion and stated they had experienced managing a CP footballer with a suspected concussion. However, several clinicians indicated that they relied on simply 'knowing the players' and assessing them subjectively, with a surprisingly high reliance on imaging despite the limited supporting evidence base. ${ }^{7}$ The use of SCAT3 (or any similar validated concussion assessment tool) was not widespread, and the reasons for this need further exploration.

When the management of concussion was discussed, a number of clinicians reported a faster return to play than advocated by the 2012 Zurich consensus statement. ${ }^{7}$ Misconceptions relating to concussion prevention, coupled with many clinicians being unaware of the existence of concussion guidelines, imply that educational interventions are indicated for this population. The majority of medical staff preferred this education to be provided through courses or online resources. ${ }^{8}$

\section{CALL TO ACTION}

The lack of cohesion evident in the variability of concussion management approaches means it is unsurprising that clinicians expressed a desire for a consensus on concussion specific to guide disability sport. While the Zurich consensus statement ${ }^{7}$ has been widely supported and adopted by many international sporting organisations, there is a clear need for more specific clinical guidance, as well as further research and education regarding the diagnosis of concussion in disability sports. This research should look to produce more normative data in disability sports, so that this clinical guidance is more specific. This work is imperative for those currently working and participating at the elite and recreational levels of disability sports, to ensure the welfare of current and future athletes. With the recent Paralympic Games being broadcast to a global audience, the profile of disability sports is higher than ever. Undertaking the process of improving concussion care in the disability sporting community will provide an opportunity to demonstrate best-practice management to those who are likely to be inspired by disability sports.

Twitter Follow Liam West @Liam_West, Steffan Griffin @lifestylemedic and Osman Ahmed @osmanhahmed

Contributors LRW and SG conceived the concept of this paper. All authors participated in discussing the ideas in this paper. LRW and SG generated the primary draft of this paper. All authors assisted in creating the final version of this paper.

\section{Competing interests None declared.}

Provenance and peer review Not commissioned; externally peer reviewed.

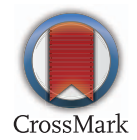

To cite West LR, Griffin S, Weiler R, et al. Br J Sports Med Published Online First: [please include Day Month Year] doi:10.1136/bjsports-2016-096767

Accepted 16 November 2016

Br J Sports Med 2016;0:1-2.

doi:10.1136/bjsports-2016-096767

\section{REFERENCES}

1 Patricios J, Makdissi M. Fifty shades of grey: concussion complexities and constructive conclusions. Br J Sports Med 2015;49:492-3.

2 Mrazik M, Dennison CR, Brooks BL, et al. . A qualitative review of sports concussion education: 


\section{Editorial}

management of acute concussions differ in specific groups? Br J Sports Med 2013;47:314-20. Br J Sports Med 2015;49:1548-53.

3 Webborn N, Cushman D, Blauwet CA, et al. The epidemiology of injuries in football at the London 2012 Paralympic Games. PM R 2015;15:1482.

4 Weiler R, Van Mechelen W, Fuller C, et al. Sport injuries sustained by athletes with disability: a systematic review. Sports Med 2016;2016;46:1141-53.

5 Makdissi M, Davis G, Jordan B, et al. Revisiting the modifiers: how should the evaluation and
6 Ahmed OH, Hussain AW, Beasley l, et al. Enhancing performance and sport injury prevention in disability sport: moving forwards in the field of football. $\mathrm{Br}$ J Sports Med 2015:49:566-7.

7 McCrory P, Meeuwisse WH, Aubry M, et al. Consensus statement on concussion in sport: the 4th International Conference on Concussion in Sport held in Zurich, November 2012. Br J Sports Med 2013:47:250-8.
8 Parker E, Gilchrist J, Schuster $D_{\text {; }}$ et al Reach and knowledge change among coaches and other participants of the online course: "concussion in sports: what you need to know". J Head Trauma Rehabil, 2015;30:198-206.

9 Weiler R, van Mechelen W, Fuller C, et al. Do neurocognitive SCAT3 baseline test scores differ between footballers living with and without disability? A cross-sectional study. Clin J Sports Med 2016; 